\title{
LETTER
}

\section{Evaluation of a protocol for resuscitation in burn patients}

\author{
Manuel Sánchez-Sánchez , Abelardo Garcia-de-Lorenzo, Eva Herrero, Beatriz Galván, Maria J Asensio and \\ Lucia Cachafeiro \\ See related research by Sánchez-Sánchez et al. http://ccforum.com/content/17/4/R176 and Berger et al. http://ccforum.com/content/17/5/195
}

We have read the commentary by Berger and Que [1] about our paper [2]. We are thankful to them for their comments; we would like to clarify some points.

First, the crude mortality rate, duration of mechanical ventilation, and renal dysfunction cannot be solely attributed to the resuscitation protocol. In addition, other factors are involved, such as intercurrent infection caused by an outbreak of multidrug-resistant Klebsiella pneumoniae strain [3].

Second, the relative initial elevation of the Sequential Organ Failure Assessment score or some intraabdominal pressure measurements $>20 \mathrm{mmHg}$ cannot be interpreted as a failure due to resuscitation.

Third, clinical practices involving surgery, hydrotherapy under sedation, and other traumatic situations often prevent an adequate supply of nutrients with enteral nutrition; this problem is also applicable to those patients whose enteral nutrition is started early. Consequently, parenteral nutrition as a supplement to enteral nutrition is often recommended in such cases [4], which is actually not total parenteral nutrition, and the use of supplementary parenteral nutrition does not reflect gut dysfunction.

Finally, the use of a resuscitation protocol for monitoring transpulmonary thermodilution parameters at below-normal levels and ensuring tissue perfusion with lactate measurements was safe and avoided overresuscitation, and was also necessary because hypovolemia might not be reflected by urine output. Future studies may reveal appropriate targets to achieve better results with this monitoring.

\section{Competing interests}

The authors declare that they have no competing interests.

\footnotetext{
* Correspondence: manuelsanchezsa@gmail.com

Unidad de Quemados Críticos, Servicio de Medicina Intensiva, Hospital

Universitario La Paz/Idipaz, Paseo de la Castellana 264, 28046 Madrid, Spain
}

Published: 20 May 2014

References

1. Berger M, Que Y: A protocol guided by transpulmonary thermodilution and lactate levels for resuscitation of patients with severe burns. Crit Care 2013, 17:195.

2. Sánchez-Sánchez M, Garcia-de-Lorenzo A, Herrero E, Lopez T, Galván B, Asensio M, Cachafeiro L, Casado C: A protocol for resuscitation of severe burn patients guided by transpulmonary thermodilution and lactate levels: a 3-year prospective cohort study. Crit Care 2013, 17:R176.

3. Sánchez M, Herruzo R, Marban A, Araujo P, Asensio M, Leyva F: Risk factors for outbreaks of multidrug-resistant Klebsiella pneumoniae in critical burn patients. J Burn Care Res 2012, 33:386-392.

4. Garcia-de-Lorenzo A, Grau T, Montejo J, Ortiz-Leyva C, Ruiz S: Complementary parenteral nutrition in the critically ill patient. Nutr Hosp 2008, 23:203-205.

$10.1186 / \mathrm{cc} 13881$

Cite this article as: Sánchez-Sánchez et al.: Evaluation of a protocol for resuscitation in burn patients. Critical Care 2014, 18:430 